\title{
Aesthetic Exploration of Modern Graphic Design
}

\author{
Limin Sui ${ }^{1, a}$ \\ ${ }^{1}$ Urumqi Vocational University, Urumqi, Xinjiang, 830002 \\ ${ }^{a}$ email
}

Keywords: Modern Graphic Design, Aesthetic Exploration, Society Research

\begin{abstract}
Traditional aesthetics contains our traditional aesthetic, modern graphic design is more fashionable international artistic expression. The two seemingly different, but in reality there is the same place of art, there can be combined with the artistic basis. China's traditional art, whether it is its form or form of expression, emphasis on the beauty and as the highest aesthetic standards. Traditional aesthetics are compatible with the idea of the formation of the natural and human society to cover the traditional aesthetic concept of modern graphic design, whether in the creative concept or in the creative techniques have a positive inspiration. Traditional aesthetics in modern graphic design has a wide range of application space and prospects, therefore, modern graphic design should be combined with traditional Chinese cultural characteristics and traditional aesthetic characteristics, the formation of Chinese characteristics with the graphic design, China's graphic design in order to gain world recognition.
\end{abstract}

\section{Introduction}

In the history of our country, many poets, painters and designers have aesthetic thoughts. They have rich aesthetics in their theory of poetry and literature, painting theory and calligraphy theory. These ideas are rich in heritage and are widely involved. Is the aesthetic of the fine works. For example, graphic designer Jin Taiqiang's has performance in the chic style, subject to the traditional Confucian aesthetics "quiet and indifferent" aesthetic influence.

Graphic design is also an art on the other hand. Different traditional arts such as poetry, painting in addition to a unique aesthetic system, and the aesthetic between the different art often affect each other. Such as painting can be found in the garden architecture art given the beauty, and garden architecture art also by poetry and so on, making them poetic. The above characteristics make modern graphic designers more extensive access to the essence of traditional aesthetic concepts, in addition to the daily exposure to the scope of the traditional process products. Ancient creation of a large number of crafts, but also showed a rich aesthetic concept. The ancestors have been able to create high-tech crafts with artistic ideas and aesthetic ideas because they followed the laws of beauty to design and produce. Modern graphic designers so attention to the design of the beauty, because the "technology almost" this concept, but also the relationship between science and art. The rapid development of contemporary technology provides favorable conditions for the creation of graphic designers. The new goal pursued by modern graphic designers is to show the aesthetic sense of science and art perfectly in the works.

\section{The Characteristics of Traditional Aesthetics}

China's traditional aesthetics pay attention to the mood and to the United States, these two points is precisely the characteristics of traditional Chinese aesthetics. First, emphasis on mood. Artistic mood refers to the situation of the blend, is the expression of the feelings, meaning, the reason and the shape of the shape, the territory of the camp, the spread of God's organic, harmonious unity. Specifically, the shape refers to the image that we can observe intuitively, and God refers to the highest state of artistic creation. Art can create a different kind of artistic beauty, requires it must be vivid, it can be said, shape and God is the premise of creating artistic conception. It is generally believed that a good work of art is not intuitive from the picture to see the shape, but deeply implied in the picture of the mood, this mood gives a huge imagination, and this has been our traditional 
artists tireless pursuit aims. This point, from the Song and Yuan Dynasties of China's landscape painting to find traces: the Song Dynasty landscape and the Yuan Dynasty landscape description of the key is not the same, the pursuit of the realm is also very different, but their common ground is in the pursuit of a taste of beauty, the beauty of mood.

Second, to and for the United States. China's ancient philosophy has always been to pay attention to harmony and harmony of man and thing I forget, the pursuit of harmony between man and nature, harmony with people and everything in harmony. We do not pay attention to the unity of the Confucianism, and seek common ground while reserving differences. This is the essence of ancient dialectics in ancient China: shades, the actual situation, yin and yang, movement, straight)) not only pay attention to the different forms and differences, pay more attention to inner harmony and unity. And grasp the different things, we talk about degree, only to grasp the degree, in order to be harmonious things together in harmony. This can be found in the history of our country that has been a multi-ethnic common development. In the face of differences in the cultural traditions of different nationalities, we can be inclusive and we can live in harmony.

\section{The Embodiment of Traditional Aesthetics in Design}

China's modern graphic designers draw on and reference to the world's outstanding graphic design concept, which to some extent, effectively promote the development of modern graphic design in China. However, in China, which has a long history and cultural heritage of the country, the traditional Chinese aesthetic concept also deeply affected the graphic designer's creative way and thinking. It is not difficult to find from the works of Chinese graphic design that the traditional aesthetic view has provided abundant nutrition to graphic designers, which has a great influence on the formation of their works and the determination of works. The domestic art designers have gradually attached importance to the traditional aesthetic concept in the use of modern graphic design, the traditional aesthetic form of expression and modern design techniques combine to express our culture more unified and harmonious. The traditional aesthetic view in the modern graphic design reflects the main form of beauty, clever ideas and ideas. The following two forms to do a brief introduction.

No matter what kind of works of art, the most important thing is to pay attention to the form of aesthetic factors, especially in the traditional and modern graphic design learn from each other, this is the pursuit of artistic works of the premise of beauty. Modern graphic design stress from the intensity of the sense of speed and sense of movement, which emphasizes the visual conflict, on the other hand, the sense of intensity in addition to the pursuit of a strong visual conflict, but also must have the form of resistance to external Showing the upward and upright sense, as well as expansion and fight for a sense of space. These aspects of modern graphic design are in the form of aesthetic and traditional aesthetic taste is very similar to a large extent. In today's multi-cultural context, the development trend of modern graphic design is the traditional aesthetic expression techniques and modern graphic design technology closely, which has a strong sense of identity. Creativity is the graphic design life and soul continuation of the necessary factors, graphic designers to improve their design level, in order to have a breakthrough in the design, it should fully understand the traditional aesthetic form, and learn to use the flexibility to find the traditional Aesthetic taste and modern graphic design of the best convergence point, this creative concept has been more and more designers recognized, modern graphic designer door is also trying to explore the balance of which.

\section{The Influence of Traditional Aesthetics in Design}

Good modern graphic design work to be people at a glance, and deeply feel the information conveyed by the works, in limited time and space under the conditions of the works to attract, leaving a deeper impression, there should be "one to When the ten "artistic effect, especially in the graphic design works can only be a point of view from the performance of reality, can not use all show life. Modern graphic design commonly used to have some characteristics of the things to show attractive mood, in the mood of things the core part of the centralized treatment, to reach a 
certain emotion can affect people. The traditional aesthetics emphasizes the balance of change, such as the combination of static and dynamic, the actual situation, black and white control, in line with the graphic design of the formal beauty of the law, but also the design of the aesthetic pursuit of the moment in the design of the concept of expression.

The artistic characteristics of modern graphic design determine the role of decorative color in the overall design, the subjective nature of the color in the graphic design, and the subjective imagination of color in the traditional aesthetic view is necessary. In addition, this kind of subjective imagination is not made, they come from the actual life, but also because of the different character and experience, etc., on the observed color will be different feelings, this difference is the traditional aesthetics of the use of color symbolic premise, In the graphic design using this color, not only in the form of its virtual, in the content because of some revelation and association is more substantial. Understanding the traditional aesthetic concept, combined with the actual situation of modern graphic design, the scientific absorption of the traditional concept of aesthetics, the development of modern graphic design is of great significance. Secondly, many of the rich aesthetic ideas of modern designers come from the traditional aesthetic view, which has a great effect on the innovative form of graphic designer design.

\section{The Application of Traditional Aesthetics in Modern Graphic Design}

The traditional thing is not outdated, old, but with the development and plasticity. Modern graphic design in its development process intends to learn from the traditional Chinese aesthetics, and achieved good artistic effect. Lv Shengzhong's little red man, Xu Bing's block word, and Jin Taiqiang's ink, are the traditional aesthetics applied to the modern graphic design of the excellent typical, are in the traditional visual language for reference, and the traditional Function, meaning and structure of the re-creation, and thus achieve the perfect combination of tradition and modern. Traditional aesthetics in modern graphic design has a wide range of application space and prospects. Chinese characters as the Chinese people emotional expression, communication tools, in our traditional culture and traditional aesthetics occupy an important position. Chinese characters have its unique structure and shape for the modern graphic design provides a very broad space. We know that our Chinese characters are graphical characters, can be intuitive to express the meaning of the expression, its pictographic features to make it with a certain degree of modern graphic design fit. Modern graphic design is often used in the design of Chinese characters, that is, the full use of Chinese characters pictographic features, and then on the basis of the pictographic in accordance with certain forms of the United States and the law of art processing, and thus convey the purpose of a strong visual impact. Modern graphic design the design of traditional Chinese characters is typical of the 2008 Beijing Olympic Games logo, it can be said that the traditional culture of creative use in modern graphic design paradigm, but also the use of Chinese characters to pass the design concept of the classic. The main part of this sign is the abstraction of the calligraphy of the calligraphy, like a man dancing with his arms, and like a person running, both the ancient and mysterious world of China to pass the world to pass warm, sincere welcome wishes, and pass The purpose of the Olympic Games is higher, faster and stronger. A simple sign of the traditional Chinese culture, traditional aesthetics and the modern Western Olympic spirit perfect combination, won the IOC President Jacques Rogge's mouth of praise.

In the traditional folk art of our country, the usual way to color is the use of color contrast, such as red and green contrast, black and white contrast, yellow and blue contrast, etc., which can be confirmed in China's wood chapel. China's traditional wood New Year paintings, the use of more yellow and purple, red and green superposition, contrast use. The use of traditional Chinese art colors to modern graphic design, will make modern graphic design received a good visual effect. In our traditional art, another typical example of the use of color is the use of ink color. Ink is through the black and white gray three kinds of superposition of the use of color, to show the Chinese painting ink lines wet and shade. Black and white gray is the graphic design visual relationship of the three colors, the three in this fit. In the graphic advertising design into the black and white bearing the content, is a very creative form of language. Design, according to the theme of the 
design to adjust the black and white gray three primary and secondary relations, you can in different designs to create a different atmosphere.

\section{Conclusion}

One is to continue to fully learn the Western graphic design theory, which is the basis for our development; the second is to learn the Western graphic design theory at the same time, to be discerned, have to be abandoned, can not copy; the contrary, should be combined with China's reality, Combined with the characteristics of traditional Chinese culture and traditional aesthetic characteristics, to achieve the combination of graphic design and traditional aesthetics. Only in this way can we form a graphic design with Chinese characteristics, and that China's graphic design can be recognized by the world.

\section{Acknowledgements}

Fund Project: World Bank Project Urumqi Vocational College Subproject "Altay Rock Painting Tourism Derivatives R \& D"

Item No.: 2016XY005

\section{References}

[1] Zheng Xiaoren. Modern graphic design of the status quo and development research [J]. Drama House, 2016 (19)

[2] Yin Qing. On how modern graphic design and traditional culture combined [J]. Popular arts, $2014(23)$

[3] Gu Huimin. The Influence of Traditional Aesthetics on Modern Graphic Design [J]. Art Education, 2015 (01)

[4] Zhao Yang. The application of traditional culture in modern graphic design [J]. Modern decoration (theory), 2015 (10)

[5] Qi Bo. The role of traditional culture in modern graphic design [J]. Youth years, 2016 (23)

[6] Wei Mingyue. Explore the application of "blank" in modern graphic design [J]. Tomorrow's fashion, 2016 (12) 